hep-th/0210272, INJE-TP-02-05

\title{
Holography in a Radiation-dominated Universe with a Positive Cosmological Constant
}

\author{
Rong-Gen Cai* \\ Institute of Theoretical Physics, Chinese Academy of Sciences, \\ P.O. Box 2735, Beijing 100080, China \\ Yun Soo Myung ${ }^{\dagger}$ \\ Relativity Research Center and School of Computer Aided Science, \\ Inje University, Gimhae 621-749, Korea
}

\begin{abstract}
We discuss the holographic principle in a radiation-dominated, closed Friedmann-Robertson-Walker (FRW) universe with a positive cosmological constant. By introducing a cosmological D-bound on the entropy of matter in the universe, we can write the Friedmann equation governing the evolution of the universe in the form of the Cardy formula. When the cosmological Dbound is saturated, the Friedmann equation coincides with the Cardy-Verlinde formula describing the entropy of radiation in the universe. As a concrete model, we consider a brane universe in the background of Schwarzschild-de Sitter black holes. It is found that the cosmological D-bound is saturated when the brane crosses the black hole horizon of the background. At that moment, the Friedmann equation coincides with the Cardy-Verlinde formula describing the entropy of radiation matter on the brane.
\end{abstract}

*e-mail address: cairg@itp.ac.cn

†e-mail address: ysmyung@physics.inje.ac.kr 


\section{INTRODUCTION}

The holographic principle is perhaps one of fundamental principles of nature, which relates a theory with gravity in $D$ dimensions to a theory without gravity in lower dimensions [1]. Although we do not yet completely understand how the hologram of gravity is realized, some beautiful examples have been found through the AdS/CFT correspondence [2].

In a seminal paper [3], E. Verlinde found a quite interesting holographic relation between the Friedmann equation describing a radiation-dominated, closed Friedmann-RobertsonWalker (FRW) universe and Cardy formula [4] describing the entropy of matter filling the universe. The radiation can be represented by a conformal field theory (CFT) with a large central charge, while the entropy for the latter can be expressed in terms of the so-called Cardy-Verlinde formula [3], a generalized form of the Cardy formula to any dimension. The Cardy-Verlinde formula was checked to hold for CFTs with AdS gravity dual, for instance, for CFTs dual to Schwarzschild-AdS black holes [3], Kerr-AdS black holes [5], hyperbolic AdS black holes and charged AdS black holes [6], Taub-Bolt AdS instanton solutions [7], and Kerr-Newman-AdS black holes [8]. Verlinde found that the Friedmann equation can be rewritten in the form of the Cardy-Verlinde formula with the help of three cosmological entropy bounds, and that when the Hubble entropy bound is saturated, the Friedmann equation coincides with the Cardy-Verlinde formula. This observation is very interesting in the sense that the Friedmann equation is a dynamic one describing geometric evolution of the universe while the Cardy-Verlinde formula is just a formula describing the number of degrees of freedom of matter in the universe. Therefore, the Verlinde's observation is indeed an interesting manifestation of the holographic principle in the cosmological setting.

By considering a moving brane universe in the background of Schwarzschild-AdS black holes in arbitrary dimensions, the holographic connection between the geometry and matter can be realized. A radiation-dominated closed FRW universe appears as an induced metric on the brane embedded in the bulk background. Savonije and Verlinde [9] interpreted the radiation as the thermal CFT dual to the bulk Schwarzschild-AdS black hole. Further they observed that when the brane crosses the bulk black hole horizon, (i) the entropy and temperature of the universe can be simply expressed in terms of the Hubble parameter and its time derivative; (ii) the entropy formula (Cardy-Verlinde formula) of CFTs in any dimension coincides with the Friedmann equation; and (iii) the Hubble entropy bound is just saturated by the Bekenstein-Hawking entropy of bulk black holes.

Since then a lot of studies have been done focusing on the generalization of [3,9] to various bulk geometries. In this paper, we are interested in the case where a positive cosmological constant is present in the universe. Namely we will discuss the holography for a radiationdominated closed FRW universe with a positive cosmological constant. This is motivated partially by the de Sitter (dS)/CFT correspondence [10], and partially by recent astronomical observations on supernova indicating that our universe is accelerating [11], which can be interpreted as that there might be a positive cosmological constant in our universe. We will start with a brief review on the case without the cosmological constant [3] in the next section, and then discuss the holography in the case with a positive cosmological constant by introducing a cosmological D-bound on the entropy of matter in the universe. As an example, in Sec. III we investigate the holographic connection between the Friedmann equation and the Cardy-Verlinde formula in the brane cosmology in the background of Schwarzschild-de 
Sitter black holes. We end this paper in Sec. IV with some conclusions and discussions.

\section{HOLOGRAPHY IN A RADIATION-DOMINATED CLOSED UNIVERSE WITH A POSITIVE COSMOLOGICAL CONSTANT}

Let us consider an $(n+1)$-dimensional closed FRW universe

$$
d s^{2}=-d \tau^{2}+R^{2} d \Omega_{n}^{2}
$$

where $R$ is the scale factor of the universe and $d \Omega_{n}^{2}$ denotes the line element of an $n$ dimensional unit sphere. The evolution of the universe is determined by the FRW equations

$$
\begin{aligned}
& H^{2}=\frac{16 \pi G_{n+1}}{n(n-1)} \frac{E}{V}-\frac{1}{R^{2}}+\frac{1}{l_{n+1}^{2}}, \\
& \dot{H}=-\frac{8 \pi G_{n+1}}{n-1}\left(\frac{E}{V}+p\right)+\frac{1}{R^{2}},
\end{aligned}
$$

where $H$ represents the Hubble parameter with the definition $H=\dot{R} / R$ and the overdot stands for derivative with respect to the cosmic time $\tau, E$ is the energy of matter filling the universe, $p$ is the pressure, $V$ is the volume of the universe, $V=R^{n} \Omega_{n}$ with $\Omega_{n}$ being the volume of an $n$-dimensional unit sphere, and $G_{n+1}$ is the Newton constant in $(n+1)$ dimensions. In addition, $l_{n+1}^{2}$ is related to the cosmological constant $\Lambda_{n+1}=n(n-1) / 2 l_{n+1}^{2}$ in $(n+1)$ dimensions.

\section{A. The case without cosmological constant}

In [3] Verlinde introduced three entropy bounds ${ }^{1}$ :

$$
\begin{aligned}
\text { Bekenstein - Verlinde entropy : } S_{\mathrm{BV}} & =\frac{2 \pi}{n} E R \\
\text { Bekenstein - Hawking entropy : } S_{\mathrm{BH}} & =(n-1) \frac{V}{4 G_{n+1} R} \\
\text { Hubble entropy : } S_{\mathrm{H}} & =(n-1) \frac{H V}{4 G_{n+1}} .
\end{aligned}
$$

The Bekenstein-Verlinde entropy bound is supposed to hold for a weakly self-gravitating universe $(H R \leq 1)$, while the Hubble entropy bound works when the universe is in the strongly self-gravitating phase $(H R \geq 1)$. In the case without the cosmological constant, the Friedmann equation [the first equation in (2.2)] can be rewritten as

\footnotetext{
${ }^{1}$ In [3] the first bound is called the Bekenstein bound. In fact this bound is slightly different from the original Bekenstein bound [12] by a numerical factor $1 / n$. So we call this the BekensteinVerlinde bound. This bound could be viewed as the counterpart of the Bekenstein bound in the cosmological setting [13].
} 


$$
S_{\mathrm{H}}=\sqrt{S_{\mathrm{BH}}\left(2 S_{\mathrm{BV}}-S_{\mathrm{BH}}\right)},
$$

in terms of those three entropy bounds. Expression (2.4) is similar to the Cardy formula [4], an entropy formula of CFTs in two dimensions. It is interesting to note that when $H R=1$, one has $S_{\mathrm{BV}}=S_{\mathrm{BH}}=S_{\mathrm{H}}$.

Let us define a quantity $E_{\mathrm{BH}}$ which corresponds to energy needed to form a black hole with size of the whole universe through the relation: $S_{\mathrm{BH}}=(n-1) V / 4 G_{n+1} R \equiv 2 \pi E_{\mathrm{BH}} R / n$. With this quantity, the Friedmann equation (2.4) can be further cast to

$$
S_{\mathrm{H}}=\frac{2 \pi R}{n} \sqrt{E_{\mathrm{BH}}\left(2 E-E_{\mathrm{BH}}\right)},
$$

which takes the same form as the Cardy-Verlinde formula [3]

$$
S=\frac{2 \pi R}{n} \sqrt{E_{c}\left(2 E-E_{c}\right)} .
$$

This formula is supposed to describe the entropy $S$ of a CFT living on an $n$-sphere of radius $R$. Here $E$ is the total energy of the CFT and $E_{c}$ stands for the Casimir energy of the system, the non-extensive part of the total energy. Now we suppose that the entropy of the radiation matter in the FRW universe can be described by the Cardy-Verlinde formula. Comparing (2.5) with (2.6), one can easily see that if $E_{\mathrm{BH}}=E_{c}, S_{\mathrm{H}}$ and $S$ must be equal. In other words, the Hubble entropy bound is saturated by the entropy of radiation matter in the universe if the Casimir energy $E_{c}$ is just enough to form a black hole with the size of the universe. At that moment, equations (2.5) and (2.6) coincide with each other. This implies that the Friedmann equation somehow knows the entropy formula of radiation-matter filling the universe [3]. Considering a brane universe in the background of Schwarzschild-AdS black holes, Savonije and Verlinde [9] found that when the brane crosses the black hole horizon, the Hubble entropy bound is saturated by the entropy of black holes in the bulk.

In Eq. (2.6) the Casimir energy $E_{c}$ is defined as [3]

$$
E_{c}=n(E+p V-T S),
$$

where $T$ stands for the temperature of the thermal CFT, $p$ is the pressure and $V$ is the volume of the system. Further Verlinde found that except for the similarity between the Friedmann equation (2.5) and the Cardy-Verlinde formula (2.6), there is also a similarity between the second equation in (2.2) concerning the time derivative of Hubble parameter and the equation (2.7) about the Casimir energy of CFTs. Let us define a (limiting) temperature

$$
T_{\mathrm{H}}=-\frac{\dot{H}}{2 \pi H} .
$$

Here the minus sign is necessary to get a positive result. In addition, it is assumed that we are in the strongly self-gravitating phase with $H R \geq 1$ so that $H \neq 0$ and $T_{\mathrm{H}}$ is well-defined. With this temperature, the second equation in (2.2) can be rewritten as

$$
E_{\mathrm{BH}}=n\left(E+p V-T_{\mathrm{H}} S_{\mathrm{H}}\right) .
$$

Thus we see that when the Hubble bound $S_{\mathrm{H}}$ is saturated by the matter entropy $S$, the (limiting) temperature $T_{\mathrm{H}}$ equals the thermodynamic temperature $T$ of matter filling the universe. Note that like the Hubble entropy bound, the (limiting) temperature $T_{\mathrm{H}}$ is a geometric quantity determined by the Hubble parameter and its time derivative. Clearly the temperature $T_{\mathrm{H}}$ is the minimal one in the strongly self-gravitating phase [3]. 


\section{B. The case with a positive cosmological constant}

Now we turn to the case with a non-vanishing cosmological constant, and generalize those interesting observations to a radiation-dominated closed FRW universe with a positive cosmological constant. We will argue that three cosmological entropy bounds in (2.3) remain the same forms even when a cosmological constant is present.

To write down a formula like (2.4), let us first discuss three entropy bounds in (2.3). The Bekenstein-Verlinde bound $S_{\mathrm{BV}}$ is the counterpart of the Bekenstein entropy bound [12] in the cosmological setting [13]. It is supposed to hold for a system with limited self-gravity, which means that the gravitational self-energy of the system is small compared to the total energy $E$. Namely, the gravitational effect on the bound can be neglected. Therefore this bound is independent of gravity theories. It is also independent of whether or not the gravity theory under consideration includes a cosmological constant. In other words, the form of the Bekenstein-Verlinde bound should remain unchanged in any gravitational theory ${ }^{2}$. Hence even when a positive cosmological constant is present, the BekensteinVerlinde bound still takes the form in (2.3). As a result, for a radiation-dominated FRW universe with a cosmological constant, the Bekenstein-Verlinde bound is a constant because of $E \sim R^{-1}$. Thus once this bound is satisfied at one time, it will be always satisfied at all times if the entropy $S$ of matter does not change.

As for the Bekenstein-Hawking bound in (2.3), it can be viewed as the holographic Bekenstein-Hawking entropy of a black hole with the size of the universe [3]. Indeed, it varies like an area instead of the volume. And for a closed universe it is the closest one that can lead to the usual area formula of black hole entropy $A / 4 G$. We know from the thermodynamics of black holes that in Einstein gravity the entropy of a black hole is always proportional to its horizon area in spite of whether or not the gravitational theory includes a cosmological constant [15]. Further, as argued by Verlinde [3], the role of $S_{\mathrm{BH}}$ is not to serve as a bound on the total entropy, but rather on a sub-extensive component of the entropy that is associated with the Casimir energy of CFTs. The above leads to the conclusion that the Bekenstein-Hawking bound should remain unchanged in its form and implication as in the case without the cosmological constant.

Finally we consider the Hubble entropy bound, which is an entropy bound for matter in a strongly self-gravitating universe $(H R \geq 1)$. In such a strongly self-gravitating universe, black holes might occur. As argued in [16,17], the maximal entropy inside the universe is produced by black holes with size of the Hubble horizon. The usual holographic argument shows that the total entropy should be less than or equal to the Bekenstein-Hawking entropy of a Hubble-horizon-sized black hole times the number of Hubble regions in the universe. In [3], by using a local holographic bound due to Fischler and Susskind [16] and Bousso [18], see also [19], Verlinde "derived" the Hubble entropy bound in (2.3). It is worth noting that in the "derivation" of the Hubble bound, Verlinde used mainly the idea that the entropy flow $S$ through a contracting light sheet is less than or equal to $A / 4 G$, where $A$ is the area of the surface from which the light sheet originates. Hence we insist that the cosmological

\footnotetext{
${ }^{2}$ In [14] we show that the Bekenstein entropy bound always has the form $S_{\mathrm{B}}=2 \pi E R$ independent of gravity theories by applying a Geroch process to an arbitrary black hole.
} 
constant will not affect the form of the Hubble bound (see also [14]). This conclusion is based on the fact that even if a cosmological constant is present, it will not occur explicitly in the "derivation" of the Hubble entropy bound.

We conclude that in a closed FRW universe with a cosmological constant, three bounds introduced in (2.3) are still applicable. That is, their forms and implications remain unchanged even if the cosmological constant is present. However, we see that the cosmological constant indeed affects the evolution of the universe. Is there a similar relation between the Friedmann equation in (2.2) and the Cardy-Verlinde formula (2.6) as in the case without the cosmological constant? Our key observation is that the positive cosmological constant provides an additional entropy measure. When the cosmological constant occurs, not the Hubble bound, but a new cosmological D-bound plays the role as the Hubble bound does in the case without the cosmological constant.

Let us go to the details. We know that in a pure de Sitter universe, there is a cosmological horizon for an inertial observer. Like a black hole horizon, the cosmological horizon has a Hawking temperature and an associated entropy [20]. The entropy is proportional to the area of the cosmological horizon. It is a geometric quantity although it has a statistical origin in quantum gravity. In an asymptotically de Sitter space, the cosmological horizon shrinks. Applying the generalized second law of thermodynamics [21] to the cosmological horizon, one can immediately find that the entropy of matter in the asymptotically de Sitter space has to be bounded above by the difference (D) between the entropy of the pure de Sitter space and the Bekenstein-Hawking entropy of the asymptotically de Sitter space:

$$
S_{\mathrm{m}} \leq \frac{1}{4 G}\left(A_{0}-A\right)
$$

where $A_{0}$ and $A$ are areas of cosmological horizons for the pure de Sitter and asymptotically de Sitter spaces, respectively. This is the so-called D-bound proposed by Bousso in [22]. The D-bound is closely related to the Bekenstein bound which applies in flat backgrounds $[22,13]$.

In our present context, the occurrence of the cosmological constant does not guarantee that the universe approaches to a de Sitter phase. When the matter is dominated, the universe behaves as the case without the cosmological constant: the universe starts from a big bang, reaches a maximal radius and then re-collapses with a big crunch. From (2.2), however, we see that for an empty flat universe ${ }^{3}$, the Hubble radius is just the cosmological horizon size $l_{n+1}$ of de Sitter space. It implies that the cosmological constant provides a new entropy measure in the universe. By analogy with the Hubble entropy bound $[16,17,3]$, we define a quantity

$$
S_{\Lambda}=(n-1) \frac{V}{4 G_{n+1} l_{n+1}} .
$$

which is the entropy of a de Sitter horizon times the number of the regions with the size of the de Sitter horizon in the universe. Like the Hubble entropy bound, it is also a geometric quantity. Together with the three entropy bounds in (2.3), the Friedmann equation in (2.2) can be rewritten as

\footnotetext{
${ }^{3}$ In that case, $E=p=0$, and the term $1 / R^{2}$ will be also absent.
} 


$$
S_{\mathrm{H}}^{2}-S_{\Lambda}^{2}=S_{\mathrm{BH}}\left(2 S_{\mathrm{BV}}-S_{\mathrm{BH}}\right)
$$

Further we note that the cosmological horizon in the asymptotically de Sitter spaces is always less that of corresponding de Sitter spaces, but one can see from (2.2) that the Hubble radius $H^{-1}$ is not always less than the cosmological horizon $l_{n+1}$ of de Sitter spaces. As a result, the left-hand side of equation (2.12) is not always positive. In addition, we stress that in the case without the cosmological constant, the Hubble bound in (2.5) is a geometric quantity, which gives an entropy bound of matter in the universe when the universe is in the strongly self-gravitating phase. Considering the D-bound (2.10) of matter in de Sitter spaces and the similarity between (2.4) and (2.12), we can define a cosmological entropy bound in the universe with a positive cosmological constant ${ }^{4}$

$$
S_{\mathrm{D}}=\sqrt{\left|S_{\mathrm{H}}^{2}-S_{\Lambda}^{2}\right|}
$$

We call it the cosmological D-bound, which can be viewed as the counterpart of the Dbound in the cosmology setting. Note that the cosmological D-bound is a square root of the difference between two geometric quantity squares, while the D-bound in de Sitter spaces is the difference between two geometric quantities. Furthermore, we would like to mention here that the implications of these two entropy bounds are quite different. The D-bound (2.10) is an entropy bound where the entropy is measurable to an inertial observer in the de Sitter space(recall the inertial observer can see only a part of the de Sitter space [20]), while the cosmological D-bound (2.13) is supposed to be an entropy bound of matter filling the whole universe with a positive cosmological constant when the universe is in the strongly selfgravitating phase. Therefore, more precisely, the cosmological D-bound should be regarded as the counterpart of the Hubble entropy bound $S_{\mathrm{H}}$ in the case with a positive cosmological constant. This can be seen clearly after comparing (2.4) and (2.12). With the choice (2.13), the Friedmann equation can be cast to the Cardy-Verlinde form even when the cosmological constant is present.

On the analogy of the (limiting) temperature $T_{\mathrm{H}}$ in $(2.8)$, we further define a new geometric temperature in our case

$$
T_{\mathrm{D}}=-\frac{\dot{H}}{2 \pi \sqrt{\left|1 / l_{n+1}^{2}-H^{2}\right|}} .
$$

Note that this is also a geometric quantity like $T_{\mathrm{H}}$ for the case without the cosmological constant. With this, the second equation in (2.2) can be expressed as

$$
E_{\mathrm{BH}}=n\left(E+p V-T_{\mathrm{D}} S_{\mathrm{D}}\right)
$$

Here the definition of $E_{\mathrm{BH}}$ is the same as the one in (2.5). Like the limiting temperature $T_{\mathrm{H}}$, the geometric temperature $T_{\mathrm{D}}$ will equal the thermodynamic temperature of matter filling

\footnotetext{
${ }^{4}$ Since a black hole larger than the cosmological horizon cannot form, one therefore should have $S_{\mathrm{H}} \geq S_{\Lambda}$. As a result, if $S_{\mathrm{H}}<S_{\Lambda}$, a cosmological singularity might occur during the evolution of the universe.
} 
the universe when the cosmological D-bound gets saturated. That is, $T_{\mathrm{D}}$ is the lower bound of thermodynamic temperature during the universe is in the strongly self-gravitating phase.

Now we turn to the Cardy-Verlinde formula (2.6). In the form (2.6) it is implicitly assumed that one has $2 E-E_{c} \geq 0$ for any CFT. In fact, in some circumstances, this condition does not get satisfied. For example, in the CFT description dual to the thermodynamics of Schwarzschild-de Sitter black hole horizon, this quantity is negative [23]. In that case, the Cardy-Verlinde formula should be changed to

$$
S=\frac{2 \pi R}{n} \sqrt{E_{c}\left(E_{c}-2 E\right)}
$$

where the definition of $E_{c}$ is still the same as the one (2.7).

In summary, for a radiation-dominated closed FRW universe with a positive cosmological constant the dynamic equations can be rewritten as

$$
\begin{aligned}
& S_{\mathrm{D}}=\frac{2 \pi R}{n} \sqrt{E_{\mathrm{BH}}\left(2 E-E_{\mathrm{BH}}\right)}, \\
& E_{\mathrm{BH}}=n\left(E+p V-T_{\mathrm{D}} S_{\mathrm{D}}\right),
\end{aligned}
$$

when $S_{\mathrm{H}} \geq S_{\Lambda}$, while the entropy of the radiation can be expressed as

$$
\begin{aligned}
& S=\frac{2 \pi R}{n} \sqrt{E_{c}\left(2 E-E_{c}\right)}, \\
& E_{c}=n(E+p V-T S) .
\end{aligned}
$$

On the other hand, when $S_{\mathrm{H}} \leq S_{\Lambda}$, the dynamic equations can be rewritten as

$$
\begin{aligned}
& S_{\mathrm{D}}=\frac{2 \pi R}{n} \sqrt{E_{\mathrm{BH}}\left(E_{\mathrm{BH}}-2 E\right)}, \\
& E_{\mathrm{BH}}=n\left(E+p V-T_{\mathrm{D}} S_{\mathrm{D}}\right),
\end{aligned}
$$

and the entropy expressions are

$$
\begin{aligned}
& S=\frac{2 \pi R}{n} \sqrt{E_{c}\left(E_{c}-2 E\right)}, \\
& E_{c}=n(E+p V-T S) .
\end{aligned}
$$

When the cosmological D-bound is saturated by the entropy $S$ of radiation matter, both sets of equations (2.17) [or (2.19)] and (2.18) [or (2.20)] coincide with each other, just like the case without the cosmological constant. The cosmological D-bound in (2.13) provides an entropy bound for matter filling the universe when the universe is in the strongly selfgravitating phase ${ }^{5}$. Namely, the cosmological D-bound plays the same role as the Hubble bound does in the case without cosmological constant.

\footnotetext{
${ }^{5}$ When the universe is in the weakly self-gravitating phase, as argued in the above, the BekensteinVerlinde bound still works well.
} 


\section{BRANE COSMOLOGY IN THE BACKGROUND OF SCHWARZSCHILD-DE SITTER BLACK HOLES}

\section{A. Thermodynamics of Schwarzschild-de Sitter black holes}

Consider an $(n+2)$-dimensional Schwarzschild-de Sitter black hole, whose line element is

$$
d s^{2}=-f(r) d t^{2}+f(r)^{-1} d r^{2}+r^{2} d \Omega_{n}^{2}
$$

Here

$$
f(r)=1-\frac{\omega_{n} M}{r^{n-1}}-\frac{r^{2}}{l_{n+2}^{2}}, \quad \omega_{n}=\frac{16 \pi G_{n+2}}{n \Omega_{n}},
$$

$M$ stands for the mass of the Schwarzschild-de Sitter black hole in the definition of Abbott and Deser [24], $G_{n+2}$ denotes the $(n+2)$-dimensional Newton constant, and $l_{n+2}$ represents the cosmological radius of the $(n+2)$-dimensional de Sitter universe. When $M=0$, the solution (3.1) reduces to a de Sitter space with a cosmological horizon at $r_{c}=l_{n+2}$. When $M$ increases from $M=0$, a black hole horizon appears and grows, while the cosmological horizon shrinks. Finally the black hole horizon $r_{\mathrm{BH}}$ touches the cosmological horizon $r_{\mathrm{CH}}$ when

$$
M=M_{N} \equiv \frac{2}{\omega_{n}(n+1)}\left(\frac{n-1}{n+1} l_{n+2}^{2}\right)^{(n-1) / 2} .
$$

This is the Nariai black hole, the maximal black hole in de Sitter space. When $M>M_{N}$, both the two horizons disappear and the solution describes a naked singularity. When $M<M_{N}$, the equation $f(r)=0$ has two real roots, the larger one is the cosmological horizon, while the smaller one is the black hole horizon.

The Hawking temperature $T_{\mathrm{HK}}$ and entropy $S$ associated with the black hole horizon are [23]

$$
T_{\mathrm{HK}}=\frac{1}{4 \pi r_{\mathrm{BH}}}\left((n-1)-(n+1) \frac{r_{\mathrm{BH}}^{2}}{l_{n+2}^{2}}\right), \quad S=\frac{r_{\mathrm{BH}}^{n} \Omega_{n}}{4 G_{n+2}} .
$$

With the identification $E=M$ and the definition $E_{c}=(n+1) E-n T_{\mathrm{HK}} S^{6}$, one can easily obtain

$$
E_{c}=\frac{2 n r_{\mathrm{BH}}^{n-1} \Omega_{n}}{16 \pi G_{n+2}}, \quad 2 E-E_{c}=-\frac{2 n r_{\mathrm{BH}}^{n+1} \Omega_{n}}{16 \pi G_{n+2} l_{n+2}^{2}} .
$$

Clearly the entropy (3.2) can be expressed by the Cardy-Verlinde formula [23]

$$
S=\frac{2 \pi l_{n+2}}{n} \sqrt{E_{c}\left(E_{c}-2 E\right)} .
$$

\footnotetext{
${ }^{6}$ Here it is assumed that the thermodynamics of the Schwarzschild-de Sitter black hole can be described in terms of a CFT. Furthermore, $E_{c}$ here is obtained from the definition (2.7) with the equation of state $p=E / n V$ of CFT's.
} 
If one rescales the energies by a factor $R / l_{n+2}$, the above equation is changed to

$$
S=\frac{2 \pi R}{n} \sqrt{E_{c}\left(E_{c}-2 E\right)} .
$$

Note that this expression is exactly the same as the entropy formula in (2.20).

\section{B. Brane dynamics in the background of Schwarzschild-de Sitter black holes}

Let us introduce an $(n+1)$-dimensional brane with tension $\sigma$ moving in the background of the Schwarzschild-de Sitter black holes (3.1). Its dynamics is determined by the following action $[9,25]$

$$
S_{\text {brane }}=\frac{1}{8 \pi G_{n+2}} \int_{\partial M} d^{n+1} x \sqrt{-h} K+\frac{1}{8 \pi G_{n+2}} \int_{\partial M} d^{n+1} x \sqrt{-h} \sigma .
$$

Here the brane is viewed as boundary of the bulk spacetime (3.1), $K$ is the extrinsic curvature for the boundary with the induced metric $h_{a b}$. Using the Israel junction equation [26], one has the equation of motion of the brane

$$
K_{a b}=\frac{\sigma}{n} h_{a b} .
$$

The brane cosmology in the Schwarzschild-de Sitter black holes has been first considered in [27]. The holography in brane cosmology in various asymptotically de Sitter spaces has also been discussed in $[27,28]^{7}$. Now let us specify the location of the brane as $r=r(t)$. We introduce a cosmic time $\tau$ so that $t=t(\tau)$ and $r=r(\tau)$ and require

$$
f(r)\left(\frac{d t}{d \tau}\right)^{2}-\frac{1}{f(r)}\left(\frac{d r}{d \tau}\right)^{2}=1
$$

which implies that the brane moves along a radial time-like geodesic in the background $(3.1)^{8}$. In that case, the induced metric $h_{a b}$ on the brane becomes

$$
d s^{2}=-d \tau^{2}+R^{2}(\tau) d \Omega_{n}^{2},
$$

which is just an $(n+1)$-dimensional closed FRW universe metric (2.1) with scale factor $R(\tau)=r(\tau)$.

Calculating the extrinsic curvature for the brane and then from the equation (3.7), we have

\footnotetext{
${ }^{7}$ However, our philosophy in understanding the holography in the case with a cosmological constant is different from those in $[27,28]$. We will discuss this point at the end of this paper.

${ }^{8}$ The dynamics of brane along a radial space-like geodesic in various asymptotically de Sitter backgrounds has also been discussed in [27,28].
} 


$$
\frac{d t}{d \tau}=\frac{\sigma R}{n f(R)}
$$

Substituting into (3.8) yields

$$
H^{2}=\frac{\omega_{n} M}{R^{n+1}}-\frac{1}{R^{2}}+\frac{1}{l_{n+2}^{2}}+\frac{\sigma^{2}}{n^{2}},
$$

The time derivative of the Hubble parameter is

$$
\dot{H}=-\frac{(n+1) \omega_{n} M}{2 R^{n+1}}+\frac{1}{R^{2}} .
$$

These equations describe a radiation-dominated closed FRW universe with a positive cosmological constant $\Lambda_{n+1}=n(n-1) / 2 l_{n+1}^{2}$ with

$$
\frac{1}{l_{n+1}^{2}}=\frac{1}{l_{n+2}^{2}}+\frac{\sigma^{2}}{n^{2}},
$$

from which we see $l_{n+1}^{2}<l_{n+2}^{2}$. Now we consider the solution of (3.11). As an example, let us discuss the special case of $n=3$. The generalization to other dimensions is straightforward. In that case, the solution has been found for three different cases depending on the parameter $\omega_{4} M / l_{n+1}^{2}$ in [29], where the authors discussed the dynamics of a non-critical brane in the Schwarzschild-AdS black hole. Defining $x=R^{2}$, we can rewrite (3.11) as

$$
\dot{x}^{2}=\frac{4}{l_{4}^{2}}\left(x-x_{+}\right)\left(x-x_{-}\right),
$$

where

$$
x_{ \pm}=\frac{l_{4}^{2}}{2}\left(1 \pm \sqrt{1-4 \omega_{4} M / l_{4}^{2}}\right) .
$$

Note that when $x=x_{ \pm}$, one has $H=0$. Actually, $x_{ \pm}$are turning points of the brane.

(1) When $4 \omega_{4} M=l_{4}^{2}$, one has $x_{+}=x_{-}$. In this case, the brane has only one turning point. However, the solution has two branches:

- $x \in\left(0, x_{+}\right]$. In this case the solution is

$$
R^{2}(\tau)=\frac{l_{4}^{2}}{2}\left(1-e^{-2 \tau / l_{4}}\right), \quad \tau \in[0, \infty)
$$

Accordingly the Hubble parameter reads

$$
H=\frac{1}{l_{4}} \frac{e^{-2 \tau / l_{4}}}{1-e^{-2 \tau / l_{4}}} .
$$

In this branch, $0 \leq H<\infty$. Since the black hole horizon $x_{\mathrm{BH}}$ falls in the range of $0<x_{\mathrm{BH}}<x_{+}$, we find $H<1 / l_{4}$ in the range of $x_{\mathrm{BH}}<x<x_{+}$. The brane trajectory is plotted in Fig. 1 as the curve (I). The brane universe starts from a big bang, reaches a maximal radius and then re-collapses to a big crunch. 
- $x \in\left[x_{+}, \infty\right)$. In this case, the solution is given by

$$
R^{2}=x(\tau)=\frac{l_{4}^{2}}{2}\left(1+e^{2 \tau / l_{4}}\right), \quad \tau \in(-\infty, \infty)
$$

And the Hubble parameter takes the expression

$$
H=\frac{1}{l_{4}} \frac{e^{2 \tau / l_{4}}}{1+e^{2 \tau / l_{4}}}
$$

Clearly in this case one has $0<H<1 / l_{4}$. The brane trajectory is plotted in Fig. 1 as the curve (II). The universe contracts with a big radius, reaches a minimal radius (the minimal radius is equal to the maximal radius in the previous branch), and then bounces to infinity. This is a singularity-free cosmology.

(2) When $4 \omega_{4} M>l_{4}^{2}$, the brane has no turning point. Namely, there is no point which has $H=0$ along the geodesic of the brane. In this case one has the solution

$$
R^{2}(\tau)=\frac{l_{4}^{2}}{2}\left(1+\sqrt{4 \omega_{4} M / l_{4}^{2}-1} \sinh \left(2 \tau / l_{4}\right)\right),
$$

where $\tau_{0} \leq \tau<\infty$ with

$$
\sinh \left(2 \tau_{0} / l_{4}\right)=-\left(4 \omega_{4} M / l_{4}^{2}-1\right)^{-1 / 2}
$$

The evolution of the Hubble parameter is given by

$$
H=\frac{1}{l_{4}} \frac{\sqrt{4 \omega_{4} M / l_{4}^{2}-1} \cosh \left(2 \tau / l_{4}\right)}{1+\sqrt{4 \omega_{4} M / l_{4}-1} \sinh \left(2 \tau / l_{4}\right)} .
$$

We find that outside the black hole horizon, $H<1 / l_{4}$. Actually, there exists another solution for the equation (3.14). But this solution describes the same movement of the brane as the solution (3.20) does. So we do not present it here. The trajectory of the brane universe is plotted in Fig. 2. In this case, the universe starts with a big bang and then expands forever.

(3) When $4 \omega_{4} M<l_{4}^{2}$, the brane has two turning points $x_{ \pm}$. The range $x \in\left[x_{-}, x_{+}\right]$is not allowed since in which $H^{2} \leq 0$. As a result, solution of equation (3.14) has two branches:

- $x \in\left(0, x_{-}\right]$. The solution is

$$
R^{2}(\tau)=\frac{l_{4}^{2}}{2}\left(1-\sqrt{1-4 \omega_{4} M / l_{4}^{2}} \cosh \left(2 \tau / l_{4}\right)\right)
$$

where $\tau$ takes value in the range $-\tau_{c} \leq \tau \leq \tau_{c}$ with

$$
\cosh \left(2 \tau_{c} / l_{4}\right)=\left(1-4 \omega_{4} M / l_{4}\right)^{-1 / 2}
$$

When $\tau= \pm \tau_{c}$, one has $R=0$ and $H=\infty$. The Hubble parameter 


$$
H=\frac{1}{l_{4}} \frac{\sqrt{1-4 \omega_{4} M / l_{4}^{2}} \sinh \left(2 \tau / l_{4}\right)}{1-\sqrt{1-4 \omega_{4} M / l_{4}^{2}} \cosh \left(2 \tau / l_{4}\right)},
$$

from which we see that $H=0$ when $\tau=0$. Note that because of the relation (3.13), it is easy to see that the black hole horizon $x_{\mathrm{BH}} \in\left(0, x_{-}\right)$, while the cosmological horizon $x_{\mathrm{CH}} \in\left(x_{+}, \infty\right)$. Thus we find that in this branch one has $H<1 / l_{4}$ as the brane stays outside the black hole horizon of the bulk background. The brane trajectory is plotted in Fig. 3 as the curve (I).

- $x \in\left[x_{+}, \infty\right)$. In this branch the solution is

$$
R^{2}(\tau)=\frac{l_{4}^{2}}{2}\left(1+\sqrt{1-4 \omega_{4} M / l_{4}^{2}} \cosh \left(2 \tau / l_{4}\right)\right), \quad \tau \in(-\infty, \infty) .
$$

And the Hubble parameter is given by

$$
H=\frac{1}{l_{4}} \frac{\sqrt{1-4 \omega_{4} M / l_{4}^{2}} \sinh \left(2 \tau / l_{4}\right)}{1+\sqrt{1-4 \omega_{4} M / l_{4}^{2}} \cosh \left(2 \tau / l_{4}\right)} .
$$

At $\tau=0$, one has $H=0$. The trajectory of the brane is plotted as the curve (II) in Fig. 3.

We conclude that the evolution of the brane depends on value of the parameter $4 \omega_{4} M / l_{4}^{2}$ and its initial position. Since we are interested in a radiation-dominated universe beginning with a big bang, so the solutions in the branch $x \in\left(0, x_{+}\right]$of case $(1)$ and in the branch $x \in\left(0, x_{-}\right]$of case (3) are suitable, respectively, for our purpose. Inspecting them, we find that $H<1 / l_{4}$ always holds when the brane stays outside the bulk black hole horizon. Further we mention that in the above discussions, the condition $4 \omega_{4} M<l_{5}^{2}$ is assumed to hold, which implies that the black hole horizon is always present.

\section{Holography in the brane cosmology}

In the brane world scenario with an AdS bulk, the tension of the brane can be adjusted to result in a so-called critical brane on which the effective cosmological constant vanishes [9]. In the present case, one can see from (3.13) that it is impossible to obtain a vanishing cosmological constant on the brane. Now we set ${ }^{9}$

$$
\sigma=n / l_{n+2}
$$

In that case the Newton constant on the brane has the relation

\footnotetext{
${ }^{9}$ For the case $\sigma \neq n / l_{n+2}$, we have a discussion in the following section. Also in that case, the relations (3.28) and (3.29) have to be changed.
} 


$$
G_{n+1}=\frac{n-1}{l_{n+2}} G_{n+2}
$$

to the Newton constant in the bulk. This relation is the same as that for a critical brane in AdS bulk [9]. Furthermore the parameter $M$ in the solution (3.1) is the black hole mass measured in the bulk coordinates [24]. According to the relation (3.10), the holographic energy $E$ measured on the brane is ${ }^{10} 11$

$$
E=\frac{l_{n+2}}{R} M
$$

Substituting (3.27), (3.28) and (3.29) into (3.11) and (3.12), we have

$$
\begin{aligned}
& H^{2}=\frac{16 \pi G_{n+1}}{n(n-1)} \frac{E}{V}-\frac{1}{R^{2}}+\frac{1}{l_{n+1}^{2}}, \\
& \dot{H}=-\frac{8 \pi G_{n+1}}{n-1}\left(\frac{E}{V}+p\right)+\frac{1}{R^{2}},
\end{aligned}
$$

with $l_{n+1}^{2}=l_{n+2}^{2} / 2$ and $p=E / n V$. These two equations are the same as the ones in (2.2). The equation of state $p=E / n V$ is just the one for radiation matter (or more general CFTs). As a result, the discussions on holography in Sec. 2 are applicable here.

Suppose the brane moves between the bulk black hole horizon and cosmological horizon ${ }^{12}$. Since the brane is viewed as the boundary of the bulk spacetime, the entropy of holographic matter (radiation) on the brane is just the entropy of black hole horizon, which is a constant during the evolution of the brane universe. However, the entropy density varies with time as

\footnotetext{
${ }^{10}$ Due to existence of the cosmological horizon in the bulk, this relation is not justified well as the case for the AdS bulk [9]. Even for the latter case, there exists a different viewpoint, for example, see [30], which argued that this relation holds only near the boundary of AdS space. However, we note that the rescaling (3.29) indeed gives a scale relation for a radiation matter in universe. Further, the relation (3.29) holds at least for small black holes as in the AdS case.
}

${ }^{11}$ In the case of AdS bulk, the boundary of the AdS space is a Lorentz spacetime, it is natural then to expect a holographic description for a dynamic brane near the boundary [9]. The boundary of dS space is a Euclidean space, however, the brane as the boundary of the bulk spacetime we have discussed so far is Lorentz, so in the spirit of dS/CFT correspondence it is very strange that there exists a holographic description for the dynamic brane in the asymptotically dS space. We thank the referee for pointing this out to us. We guess that this might be related to that the horizon thermodynamics has a universal 2-dimensional CFT description [31]. For example, for asymptotically non-AdS spaces, there is also a holographic description for a dynamic brane and the resulting equation of motion can also be cast to the Cardy-Verlinde form [32]. Therefore the holographic connection between the dynamics of the brane and the bulk horizon thermodynamics might be beyond the AdS/CFT and dS/CFT correspondences.

\footnotetext{
${ }^{12}$ Note that the brane will not cross the cosmological horizon in the case where the radiation is dominated, see the previous subsection.
} 


$$
s \equiv \frac{S}{V}=\frac{r_{\mathrm{BH}}^{n}}{4 G_{n+2} R^{n}}=\frac{(n-1) r_{\mathrm{BH}}^{n}}{4 G_{n+1} l_{n+2} R^{n}}
$$

and the energy density of radiation-matter

$$
\rho \equiv \frac{E}{V}=\frac{n r_{\mathrm{BH}}^{n-1} l_{n+2}}{16 \pi G_{n+2} R^{n+1}}\left(1-\frac{r_{\mathrm{BH}}^{2}}{l_{n+2}^{2}}\right),
$$

in terms of the black hole horizon radius $r_{\mathrm{BH}}$. Further from the scaling relation (3.29), the temperature $T$ on the brane is given by

$$
T=\frac{l_{n+2}}{R} T_{\mathrm{HK}}=\frac{l_{n+2}}{4 \pi r_{\mathrm{BH}} R}\left((n-1)-(n+1) \frac{r_{\mathrm{BH}}^{2}}{l_{n+2}^{2}}\right) .
$$

Applying the first law of thermodynamics to the radiation matter in the brane universe, one has

$$
T d s=d \rho+n(\rho+p-T s) \frac{d R}{R} .
$$

Following [9] and defining

$$
\gamma=\frac{n}{2}(\rho+p-T s) R^{2}
$$

we have

$$
\gamma=\frac{n r_{\mathrm{BH}}^{n-1} l_{n+2}}{16 \pi G_{n+2} R^{n-1}}=\frac{n(n-1) r_{\mathrm{BH}}^{n-1}}{16 \pi G_{n+1} R^{n-1}} .
$$

Obviously, the non-vanishing $\gamma$ is the effect of spatial curvature $1 / R^{2}$. That is, because of the spatial curvature, the energy of thermodynamics system has a non-extensive part. Otherwise, the quantity $\gamma$ must vanish due to the Euler relation. From (3.32), one can see that $\gamma$ is just the first term of the energy density (3.32) divided by $1 / R^{2}$. Further one can find that the entropy density (3.31) can be expressed as

$$
s=\frac{4 \pi}{n} \sqrt{\gamma\left(\frac{\gamma}{R^{2}}-\rho\right)} .
$$

Now we consider a special moment that the brane crosses the bulk black hole horizon. On that time, one has $R=r_{\mathrm{BH}}$. From (3.11) we see

$$
H^{2}=\frac{1}{l_{n+2}^{2}} .
$$

Note that we have taken $\sigma^{2} / n^{2}=1 / l_{n+2}^{2}$. At that moment the cosmological D-bound in (2.13) turns out to be

$$
S_{\mathrm{D}}=\frac{(n-1) r_{\mathrm{BH}}^{n} \Omega_{n}}{4 G_{n+1} l_{n+2}}=\frac{r_{\mathrm{BH}}^{n} \Omega_{n}}{4 G_{n+2}},
$$


which is just the black hole horizon entropy (3.2). That is, when the brane crosses the bulk black hole horizon, the cosmological D-bound is saturated by the entropy of the bulk black hole. At that time, the geometric temperature in (2.14) is given by

$$
T_{\mathrm{D}}=\frac{l_{n+2}}{4 \pi r_{\mathrm{BH}}^{2}}\left((n-1)-(n+1) \frac{r_{\mathrm{BH}}^{2}}{l_{n+2}^{2}}\right),
$$

which equals the temperature (3.33) of radiation filling the universe. Thus, we find that when the brane crosses the black hole horizon, the FRW equations (2.19) coincide with the equations (3.37) and (3.36) which describe the entropy of radiation matter in the brane universe with a positive cosmological constant. Thus we reach the same conclusion as in the case without the cosmological constant [9].

\section{CONCLUSION AND DISCUSSION}

We have discussed the holography in a radiation-dominated, closed FRW universe with a positive cosmological constant. By introducing the cosmological D-bound (2.13) on the entropy of matter in the universe, the Friedmann equation describing the evolution of the universe can be rewritten in the form of Cardy-Verlinde formula which describes the degrees of freedom of radiation matter filling the universe. When the cosmological D-bound is saturated by the entropy of matter, these two equations coincide with each other. Thus we have successfully generalized interesting observations by Verlinde $[3,9]$ on the holographic connection between the Friedmann equation and Cardy-Verlinde formula to the case with a positive cosmological constant. By considering a brane universe in the background of Schwarzschild-de Sitter black holes, we have found that the cosmological D-bound is saturated when the brane crosses the black hole horizon in the bulk background. At that moment, the Friedmann equation and Cardy-Verlinde formula coincide with each other, and the introduced geometric temperature $T_{\mathrm{D}}$ in (2.14) equals the thermodynamic temperature $T$ of the radiation matter.

We stress that when discussing the holographic connection in the brane universe in the Schwarzschild-de Sitter black holes, we have taken a special tension (3.27), which is the same as in the case for critical brane in the AdS bulk. Only in that case, the Friedmann equation and Cardy-Verlinde formula coincide with each other very well. We point out here that if the brane tension is arbitrary, the Friedmann equation still has a form as the CardyVerlinde formula, but a factor $\sigma l_{n+2} / n$ will appear in (3.37). Furthermore, for the radiation matter dual to the black holes in de Sitter spaces, we see from (3.37) that $\rho-\gamma / R^{2}<0$. If considering a non-critical brane cosmology in the Schwarzschild-AdS black holes, one will see that in that case $\rho-\gamma / R^{2}>0$. This case corresponds to the holographic connection described by equations (2.17) and (2.18). In addition, if the cosmological constant becomes negative, the minus sign in front of $S_{\Lambda}$ has to be changed to plus. The quantity (2.11) then will lose its interpretation, but all formulas will still work well.

We have noticed that the holography was discussed in many literatures in the case with a cosmological constant, for example, see [27,28,30,33-36]. However, our understanding is different from those in existing literature: in some papers the cosmological constant term is incorporated to the Bekenstein-Verlinde entropy bound; in some papers this term is kept as 
an independent term. In those literature the Friedmann equation and the Cardy-Verlinde formula have not a same form, and when the Hubble bound is saturated, these two formulas do not get matched. Finally we point out that at the end of paper [3], Verlinde mentioned that when the cosmological constant does not vanish, the Hubble entropy bound needs to be modified by replacing $H$ with the square root of $H^{2}-1 / l_{n+1}^{2}$. But, as argued in this paper, three entropy bounds: Bekenstein-Verlinde bound, Bekenstein-Hawking bound and Hubble bound in (2.3) still have the same forms as the case without the cosmological constant, even when the cosmological constant is present. The cosmological D-bound introduced in this work provides a new entropy bound of matter in the strongly self-gravitating universe

$(H R>1)$ with a positive cosmological constant and makes all formulas work so nicely as the case without the cosmological constant.

\section{ACKNOWLEDGMENT}

We thank X.J. Wang for help in drawing the figures. R.G.C. is grateful to Relativity Research Center and School of Computer Aided Science, Inje University for warm hospitality during his visit. The work of R.G.C. was supported in part by a grant from Chinese Academy of Sciences and a grant from Ministry of Education, PRC, and by the Ministry of Science and Technology of China under grant No. TG1999075401. Y.S.M. acknowledges partial support from the KOSEF grant, Project Number: R02-2002-000-00028-0. 


\section{REFERENCES}

[1] G. 't Hooft, arXiv:gr-qc/9310026; L. Susskind, J. Math. Phys. 36, 6377 (1995) [arXiv:hep-th/9409089].

[2] J. M. Maldacena, Adv. Theor. Math. Phys. 2, 231 (1998) [Int. J. Theor. Phys. 38, 1113 (1999)] [arXiv:hep-th/9711200]; S. S. Gubser, I. R. Klebanov and A. M. Polyakov, Phys. Lett. B 428, 105 (1998) [arXiv:hep-th/9802109]; E. Witten, Adv. Theor. Math. Phys. 2, 253 (1998) [arXiv:hep-th/9802150].

[3] E. Verlinde, arXiv:hep-th/0008140; "CFT\&FRW", Prepared for ICTP Spring School on Superstrings and Related Matters, Trieste, Italy, 2-10 Apr 2001. Published in *Trieste 2001, Superstrings and related matters* 79-102.

[4] J. L. Cardy, Nucl. Phys. B 270, 186 (1986).

[5] D. Klemm, A. C. Petkou and G. Siopsis, Nucl. Phys. B 601, 380 (2001) [arXiv:hepth/0101076].

[6] R. G. Cai, Phys. Rev. D 63, 124018 (2001) [arXiv:hep-th/0102113].

[7] D. Birmingham and S. Mokhtari, Phys. Lett. B 508, 365 (2001) [arXiv:hep-th/0103108].

[8] J. l. Jing, Phys. Rev. D 66, 024002 (2002) [arXiv:hep-th/0201247].

[9] I. Savonije and E. Verlinde, Phys. Lett. B 507, 305 (2001) [arXiv:hep-th/0102042].

[10] A. Strominger, JHEP 0110, 034 (2001) [arXiv:hep-th/0106113].

[11] A. G. Riess et al. [Supernova Search Team Collaboration], Astron. J. 116, 1009 (1998) [arXiv:astro-ph/9805201]; S. Perlmutter et al. [Supernova Cosmology Project Collaboration], Astrophys. J. 483, 565 (1997) [arXiv:astro-ph/9608192]; R. R. Caldwell, R. Dave and P. J. Steinhardt, Phys. Rev. Lett. 80, 1582 (1998) [arXiv:astro-ph/9708069]; P. M. Garnavich et al., Astrophys. J. 509, 74 (1998) [arXiv:astro-ph/9806396].

[12] J. D. Bekenstein, Phys. Rev. D 23, 287 (1981).

[13] R. G. Cai, Y. S. Myung and N. Ohta, Class. Quant. Grav. 18, 5429 (2001) [arXiv:hepth/0105070].

[14] R. G. Cai and Y. S. Myung, Phys. Lett. B 559, 60 (2003) [arXiv:hep-th/0210300].

[15] R. M. Wald, Phys. Rev. D 48, 3427 (1993) [arXiv:gr-qc/9307038]. In higher derivative gravitational theories, the so-called area theorem no longer holds, for example, see T. Jacobson and R. C. Myers, Phys. Rev. Lett. 70, 3684 (1993) [arXiv:hep-th/9305016]; R. G. Cai, Phys. Rev. D 65, 084014 (2002) [arXiv:hep-th/0109133]; and references therein.

[16] W. Fischler and L. Susskind, arXiv:hep-th/9806039.

[17] R. Easther and D. A. Lowe, Phys. Rev. Lett. 82, 4967 (1999) [arXiv:hep-th/9902088]; G. Veneziano, Phys. Lett. B 454, 22 (1999) [arXiv:hep-th/9902126]; G. Veneziano, arXiv:hep-th/9907012; R. Brustein and G. Veneziano, Phys. Rev. Lett. 84, 5695 (2000) [arXiv:hep-th/9912055]; D. Bak and S. J. Rey, Class. Quant. Grav. 17, L83 (2000) [arXiv:hep-th/9902173]; N. Kaloper and A. D. Linde, Phys. Rev. D 60, 103509 (1999) [arXiv:hep-th/9904120].

[18] R. Bousso, JHEP 9907, 004 (1999) [arXiv:hep-th/9905177]; R. Bousso, JHEP 9906, 028 (1999) [arXiv:hep-th/9906022].

[19] E. E. Flanagan, D. Marolf and R. M. Wald, Phys. Rev. D 62, 084035 (2000) [arXiv:hepth/9908070].

[20] G. W. Gibbons and S. W. Hawking, Phys. Rev. D 15, 2738 (1977).

[21] J. D. Bekenstein, Phys. Rev. D 9 (1974) 3292. 
[22] R. Bousso, JHEP 0104, 035 (2001) [arXiv:hep-th/0012052].

[23] R. G. Cai, Nucl. Phys. B 628, 375 (2002) [arXiv:hep-th/0112253]; R. G. Cai, Phys. Lett. B 525, 331 (2002) [arXiv:hep-th/0111093].

[24] L. F. Abbott and S. Deser, Nucl. Phys. B 195, 76 (1982).

[25] P. Kraus, JHEP 9912, 011 (1999) [arXiv:hep-th/9910149]; D. Ida, JHEP 0009, 014 (2000) [arXiv:gr-qc/9912002].

[26] W. Israel, Nuovo Cim. B 44S10, 1 (1966) [Erratum-ibid. B 48, 463 (1967 NUCIA,B44,1.1966)].

[27] S. Ogushi, Mod. Phys. Lett. A 17, 51 (2002) [arXiv:hep-th/0111008]; S. Nojiri and S. D. Odintsov, JHEP 0112, 033 (2001) [arXiv:hep-th/0107134].

[28] A. J. Medved, arXiv:hep-th/0111182; A. J. Medved, Class. Quant. Grav. 19, 919 (2002) [arXiv:hep-th/0111238]; Y. S. Myung, Phys. Lett. B 531, 1 (2002) [arXiv:hepth/0112140]; S. Nojiri, S. D. Odintsov and S. Ogushi, arXiv:hep-th/0205187.

[29] A. C. Petkou and G. Siopsis, JHEP 0202, 045 (2002) [arXiv:hep-th/0111085].

[30] A. Padilla, Phys. Lett. B 528, 274 (2002) [arXiv:hep-th/0111247]; J. P. Gregory and A. Padilla, Class. Quant. Grav. 19, 4071 (2002) [arXiv:hep-th/0204218]; Y. S. Myung, arXiv:hep-th/0208086.

[31] S. Carlip, Phys. Rev. Lett. 82, 2828 (1999) [arXiv:hep-th/9812013]; Class. Quant. Grav. 16, 3327 (1999) [arXiv:gr-qc/9906126].

[32] R. G. Cai and Y. Z. Zhang, Phys. Rev. D 64, 104015 (2001) [arXiv:hep-th/0105214].

[33] B. Wang, E. Abdalla and R. K. Su, Phys. Lett. B 503, 394 (2001) [arXiv:hepth/0101073]; B. Wang, E. Abdalla and R. K. Su, Mod. Phys. Lett. A 17, 23 (2002) [arXiv:hep-th/0106086].

[34] S. Nojiri, O. Obregon, S. D. Odintsov, H. Quevedo and M. P. Ryan, Mod. Phys. Lett. A 16, 1181 (2001) [arXiv:hep-th/0105052].

[35] D. Youm, brane universe," arXiv:hep-th/0111276.

[36] A. J. Medved, arXiv:hep-th/0112009. 


\section{FIGURES}

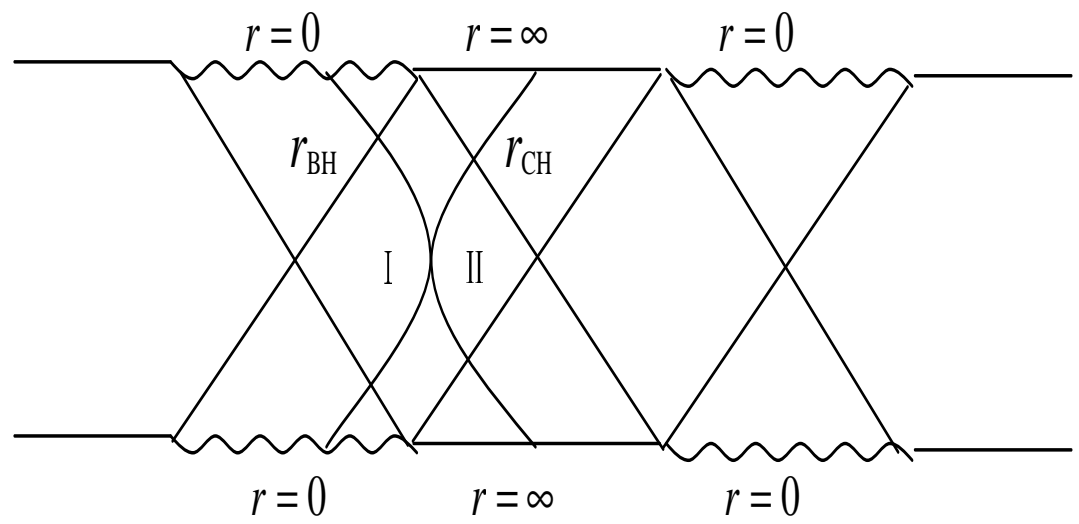

FIG. 1. The Penrose diagram of the Schwarzschild-de Sitter space. The curve (I) describes the trajectory of brane for the branch $x \in\left(0, x_{+}\right]$, and the curve (II) for the branch $x \in\left[x_{+}, \infty\right)$.

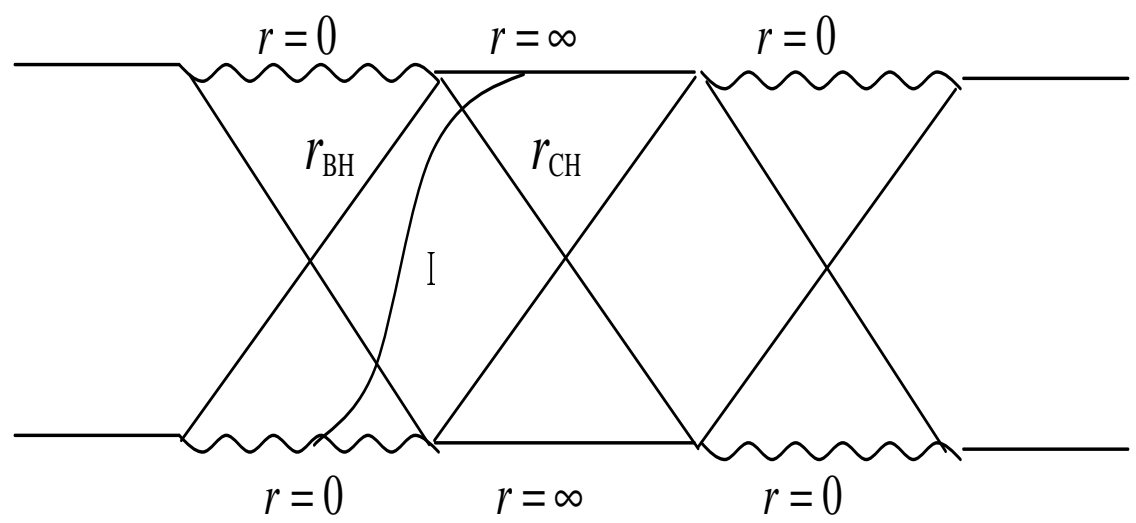

FIG. 2. The Penrose diagram of the Schwarzschild-de Sitter space. The curve (I) describes the trajectory of brane in the case $4 \omega_{4} M<l_{4}^{2}$.

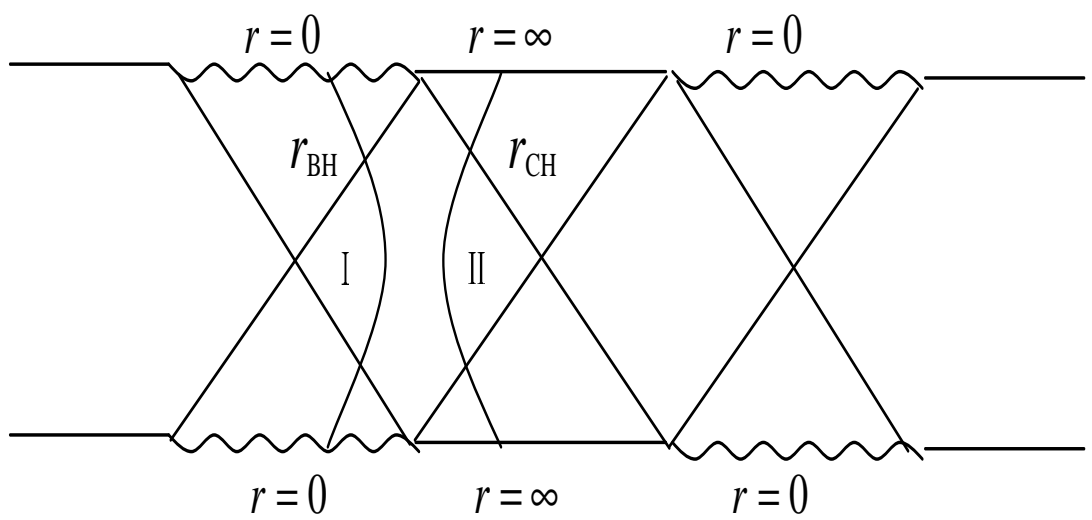

FIG. 3. The Penrose diagram of the Schwarzschild-de Sitter space. The curve (I) describes the trajectory of brane for the branch $x \in\left(0, x_{-}\right]$, and the curve (II) for the branch $x \in\left[x_{+}, \infty\right)$. 